\title{
Numerical Simulation of Bolide Entry with Ground Footprint Prediction
}

\author{
Michael J. Aftosmis* \\ NASA Ames, Moffett Field, CA \\ Donovan L. Mathias ${ }^{\ddagger}$ \\ NASA Ames, Moffett Field, CA
}

\author{
Marian Nemec ${ }^{\dagger}$ \\ Science 8 Tech. Corp., Moffett Field, CA \\ Marsha J. Berger ${ }^{\S}$ \\ NYU, 251 Mercer St., NY, NY
}

\begin{abstract}
As they decelerate through the atmosphere, meteors deposit mass, momentum and energy into the surrounding air at tremendous rates. Trauma from the entry of such bolides produces strong blast waves that can propagate hundreds of kilometers and cause substantial terrestrial damage even when no ground impact occurs. We present a new simulation technique for airburst blast prediction using a fully-conservative, Cartesian mesh, finitevolume solver and investigate the ability of this method to model far-field propagation over hundreds of kilometers. The work develops mathematical models for the deposition of mass, momentum and energy into the atmosphere and presents verification and validation through canonical problems and the comparison of surface overpressures, and blast arrival times with actual results in the literature for known bolides. The discussion also examines the effects of various approximations to the physics of bolide entry that can substantially decrease the computational expense of these simulations. We present parametric studies to quantify the influence of entry-angle, burst-height and other parameters on the ground footprint of the airburst, and these values are related to predictions from analytic and handbook-methods.
\end{abstract}

Keywords: atmospheric entry, blast propagation, meteor, asteroid, bolide, Cartesian, Cart3D

\section{Introduction}

T $^{\mathrm{N}}$ mid February 2013 an asteroid measuring approximately 20 meters in diameter entered the sky over Chelyabinsk Russia. The bolide had a mass of about 12,500 metric tons and vaporized nearly completely above $25 \mathrm{~km}$. Despite its relatively small size, the asteroid carried with it tremendous kinetic energy and deposited approximately 520 kilotons into the atmosphere as it entered and burned up. ${ }^{1}$ Over a minute later, the shock wave from this sudden deposition of energy reached the ground breaking glass and damaging structures in a region covering more than 5000 square kilometers.

Government infrasound monitoring records an average of around 27 encounters with objects larger than a meter in diameter annually. ${ }^{2}$ Each decade, approximately seven encounters occur which release over 10 kilotons into the atmosphere. ${ }^{\text {a3 }}$ Since the population of potentially hazardous objects follows a rough power law, the best current estimates predict encounters with Chelyabinsk-sized objects approximately every 80 years. $^{4}$

In response to this threat, NASA's Near-Earth Object program initiated a new research activity actively focused on Planetary Defense in October of 2014. ${ }^{5}$ This program is structured around four thrusts: (1) Characterization and Composition, (2) Entry/Break-up physics, (3) Atmospheric Propagation and Impact Effects, and (4) Physics-Based Risk Assessment.

The simulation and modeling effects discussed in the proposed paper target the third of these tasks, namely propagation of the airburst through the atmosphere and estimation of the impact effects. Clearly

*michael.aftosmis@nasa.gov, Applied Modeling and Simulation Branch, Associate Fellow AIAA.

†marian.nemec@nasa.gov, Applied Modeling and Simulation Branch, Senior Member AIAA.

$¥$ donovan.mathias@nasa.gov, Engineering Risk Assessment Team, Senior Member AIAA.

§berger@cims.nyu.edu, Courant Institute, NYU, Senior Member AIAA.

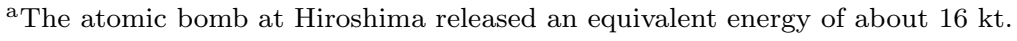


this element is closely connected to both entry/breakup and risk assessment. In a nutshell, atmospheric propagation takes inputs from the entry modeling task and computes the land/water footprint to feed risk assessment. From a simulation and modeling standpoint, modeling entry physics typically involves highly-detaled multi-physics simulations done in the frame of the asteroid as it enters, ${ }^{6}$ while atmospheric propagation is usually done in the Eulerian frame of a fixed observer on the ground and taking energydeposition as input.

In the literature, surface impact affects are often assessed using either analytic models or by modeling the energy release through correlation with static nuclear detonations. ${ }^{7-9}$ More detailed analysis has also been performed using numerical simulation of the atmospheric blast propagation using various hydrocodes. ${ }^{1,3,6,10,11}$ Such simulations are often driven by either triggered breakup of a known object or by an estimate of the energy deposition profile which can be generated for specific bolide entries through observation of the light-curve, ${ }^{1}$ or infrasound measurements. ${ }^{3,12}$

The approach taken in the proposed paper uses the energy deposition profile as input and performs blast propagation using a fully-conservative finite-volume method with adaptive meshing. Rather than reconstruct a specific entry event, our goal in this work is to drive physics-based risk assessment. To this end, we desire the flexibility to drive simulation from outputs of entry-modeling task, analytic or PDE-based models, ${ }^{7,8,13-15}$ observed light curve data, or infrasound measurements.

The final paper will include a theoretical section showing how energy deposition profiles are used to determine initial conditions for simulations taking energy deposition profiles as input. Details of the timedependent modeling approach will be presented along with a discussion of computational expense. It will also include verification and validation data using a spherical blast model at relevant conditions. Investigations will include examination of the influence of entry-angle, burst-height and other parameters. Further validation will include comparisons with data from the Chelyabinsk meteor examining both ground over-pressure and blast arrival time using data from ground reports and time-synched video. Notional titles for sections of the final paper are shown below along with some preliminary results for some of the investigations.

\section{Theoretical Development and Numerical Method}

\section{A. Conservation of Mass, Momentum, and Energy}

During its violent entry, a meteoroid transfers mass, momentum and energy into the surrounding air at tremendous rates. Observational data and models of the physical processes are frequently processed to express the net effects of the near-field physics as energy deposition profiles in terms of energy-released as functions of both time and altitude. From the point of view of a fixed computation in an Eulerian frame on the ground, this deposition can be modeled as a time-dependent source for the state of the fluid in a control volume surrounding the trajectory of the entering body.

To aid in analysis, Figure 1 shows a tube-like control volume sketched around the trajectory of a body passing through the atmosphere at high speed....

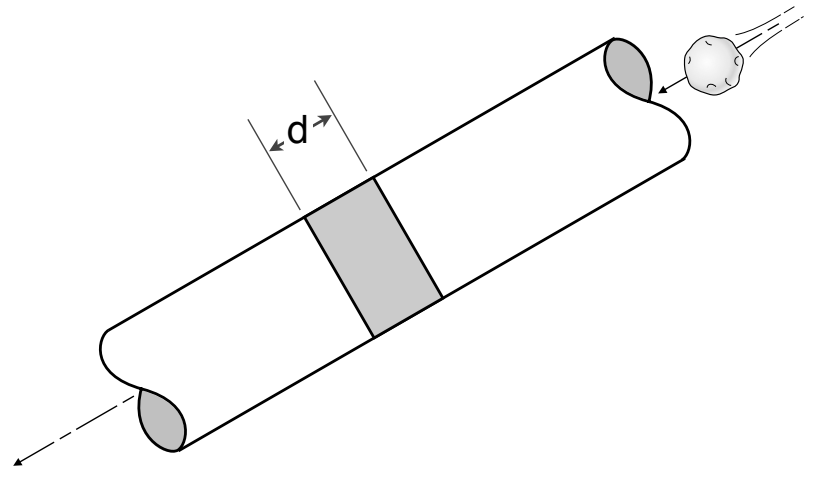

Figure 1. Sketch showing control volume around trajectory of entering body used for converting energydeposition profile data into time-dependent source terms for fluid state used in numerical simulations.

...include theoretical development to support derivation of time-dependent source terms for state variables used to drive simulations from energy-deposition profiles...

\section{B. Numerical Method}

Simulations for this work use a solver based on the Cartesian cut-cell approach of Reference [16] in which the governing equations are discretized on a multilevel Cartesian mesh with embedded boundaries. The mesh consists of regular Cartesian hexahedra everywhere, except for a layer of body-intersecting cut-cells which 
intersect the geometry at boundaries. The spatial discretization uses a second-order accurate finite volume method with a weak imposition of boundary conditions, resulting in a system of equations

$$
R\left(Q_{H}\right)=0
$$

The flux-vector splitting approach of van Leer $^{17}$ is used for residual evaluation. Although it consists of nested Cartesian cells, the mesh is viewed as an unstructured collection of control volumes making the approach well-suited for solution-adaptive mesh refinement. Steady-state flow solutions are obtained using a five-stage Runge-Kutta scheme with local time stepping and multigrid. Domain decomposition via space-

filling curves permits parallel computation; for more details see Aftosmis et al. and Berger et al. ${ }^{18-20}$

...report modification of basic flow solver to include buoyancy effects through the Froude number and discussion of atmosphere model used in numerical investigations...

\section{Meshing and Computational Resources}

\section{Numerical Investigations}

\section{A. Verification for a Strong Spherical Blast}

...Report verification results for numerical investigation of spherical blast using a spherical charge of known strength compared with analytic solution of a equivalent blast emanating from a point source. Include a comparison of predicted overpressure with results from Friedlander equation. Demonstration to show numerical method predicts well-known logarithmic asymptotic deceleration of blast wave as the radius of the blast expands...

\section{B. Chelyabinsk Meteor}

...Present validation of simulation methodology using data collected for Chelyabinsk Meteor. Use energy deposition profile from Brown et al. ${ }^{3}$ and compare with data for ground overpressure and shock arrival-time found in Reference [1]...

Figure 2 shows simulation results for Chelyabinsk meteor using deposition profile from Reference [3]. These simulations used an entry angle of $18^{\circ}$, with peak brightness near the half-energy height at $29.5 \mathrm{~km}$ altitude and a total energy of $520 \mathrm{kt}$. The computational domain was $80 \mathrm{~km}$ high and extended $256 \mathrm{~km}$ in both directions along the ground plane. The simulation included a bit over $100 \mathrm{~km}$ of the terminal flight path. The computational mesh used $\sim 90 \mathrm{M}$ cells with roughly $1 \mathrm{~m}$ resolution along the trajectory and $\sim 10 \mathrm{~m}$ on the ground in the region of peak overpressure.

Figure 3 shows the ground overpressure footprint predicted by this simulation with an overlay of the entry trajectory as seen from above. The origin of the plot on the left is at the location of peak brightness. Isobars in both frames are have an increment of $1 \%$ of ambient pressure (sea level, standard atmosphere). The map at the right shows the predicted ground-footprint overlaid onto glass damage data reported by Popova and Jenniskens et al. ${ }^{1}$ Statistical correlations in Mannan and Lees $^{21}$ for blast-wave data indicate that an overpressure of $\sim 700 \mathrm{~Pa}(0.69 \%)$ is able to shatter about $5 \%$ of typical glass windows while $\Delta p$ of $\sim 6 \%$ breaks roughly $90 \%$. The predicted overpressure contours shown in Figure 3 show very good correlation with glass damage reports.

Figure 4 shows predicted shock-wave arrival time at ground level for comparison to data in Reference [1]. The time increment between successive contours is $\Delta t=33.2$ seconds. Peak brightness is at the origin. The map in the frame at the right shows the ground track for correlating with other simulations and collected data.

\section{C. $90^{\circ}$ Entry Angle}

Risk assessment is generally performed using monte-carlo simulations of static airbursts varying both the altitude and strength of the detonation. ${ }^{22}$ This allows handbook methods, like those in Reference [9], to be 

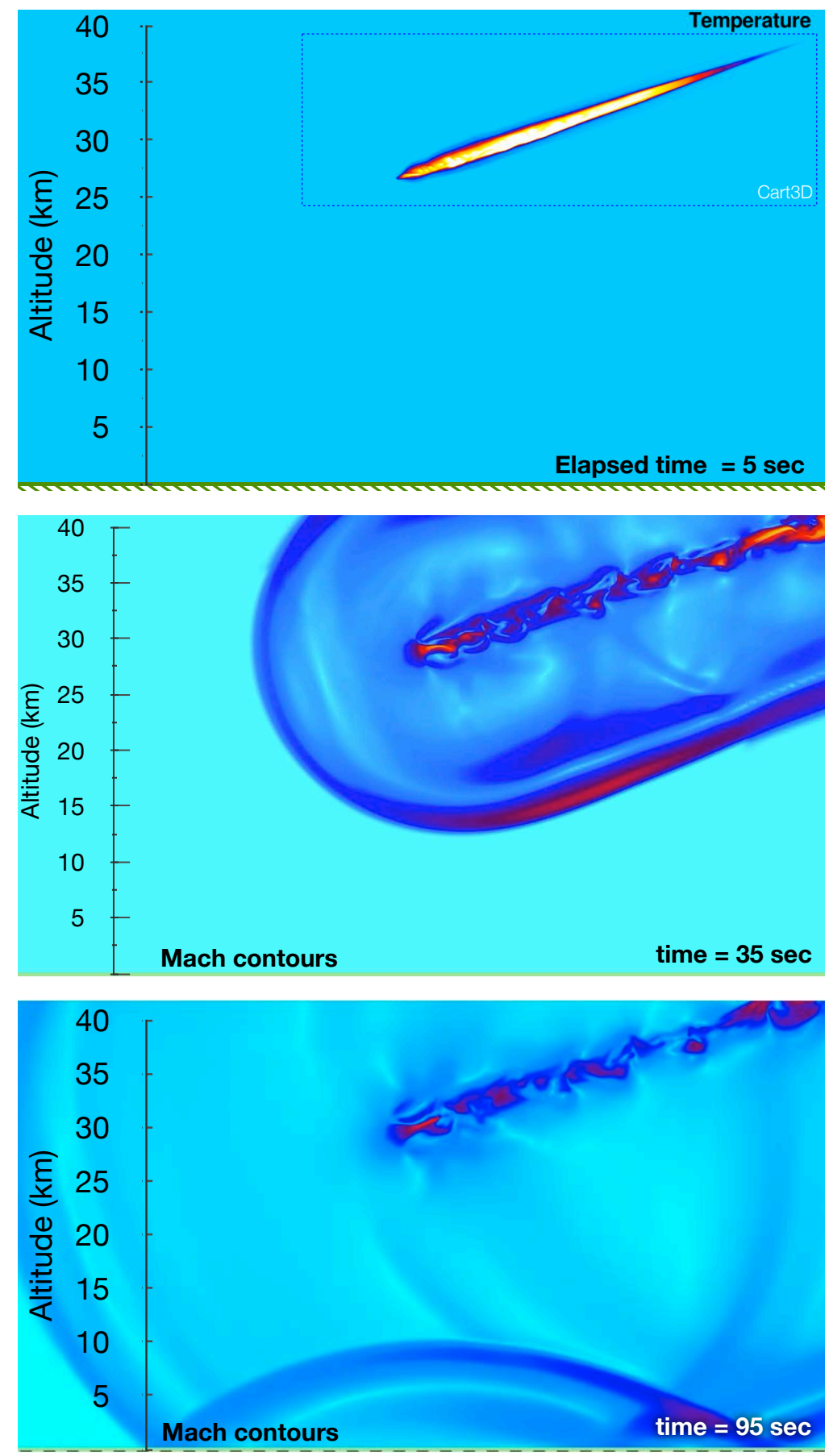

Figure 2. Simulation results for Chelyabinsk meteor using deposition profile from Brown et al. ${ }^{3} 18^{\circ}$ entry angle, with peak brightness at $29.5 \mathrm{~km}$ altitude. Total Energy $=520 \mathrm{kt}$, 

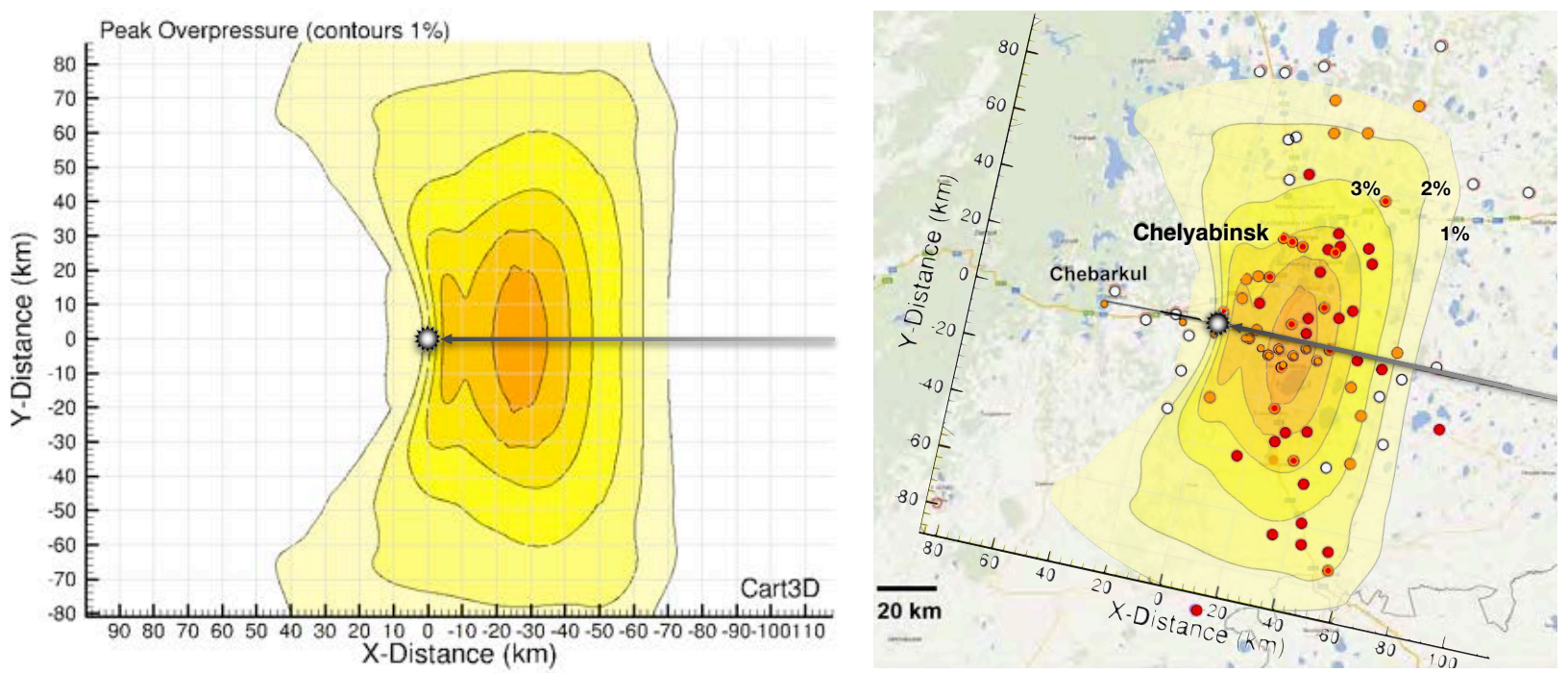

Figure 3. Ground footprint for Chelyabinsk meteor showing computed peak over-pressures overlaid on grounddamage map of Reference [1] using the energy deposition profile from Brown et al. ${ }^{3}$ Increment between isobars is $1 \%$ of sea level pressure for standard atmosphere. Peak brightness is at $(0,0)$. Statistical correlations in Mannan and Lees ${ }^{21}$ for blast-wave data indicate that an overpressure of $\sim 700 \mathrm{~Pa}(0.69 \%)$ is able to shatter about $5 \%$ of typical glass windows while $\Delta p$ of $\sim 6 \%$ breaks roughly $90 \%$.
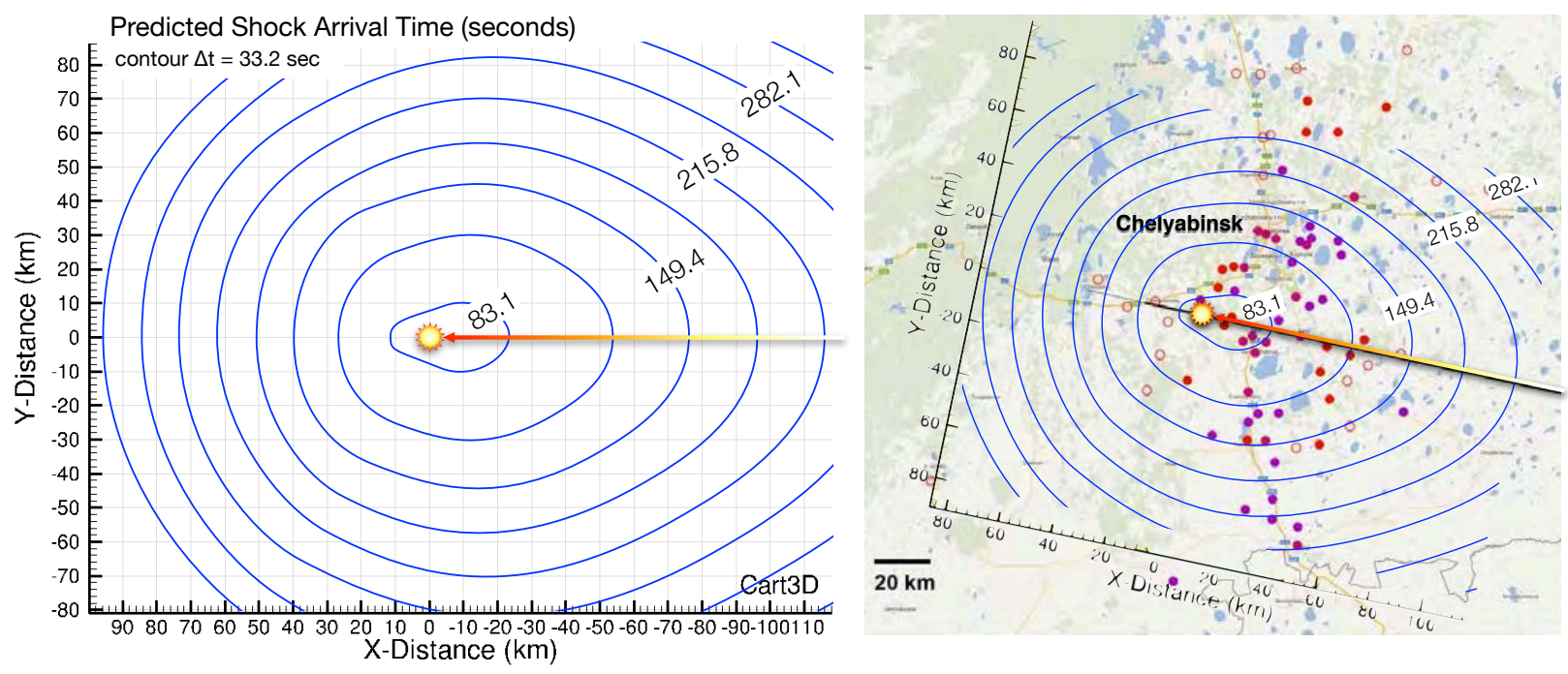

Figure 4. Ground footprint showing computed shock-wave arrival time at ground level. Map at right shows ground-track. Peak brightness is at $(0,0)$. 
used to predict ground damage with statistical correlations. As we transition our risk assessment to physicsbased blast predictions, the $90^{\circ}$ entry case is of particular interest. Symmetry dictates that at this entry angle, the blast footprint will consist of a series of concentric circles describing variation in peak overpressure with distance from ground-zero. Since the energy deposition profile is roughly logarithmic with altitude, the vast majority of the energy along the trajectory is released near the point of peak brightness. Given these observations, its interesting to compare the ground overpressures from simulations along a $90^{\circ}$ entry trajectory with those from a spherical detonation of the same magnitude at the same altitude. This case provides an important bridge between the detailed simulation of entry trajectories with more traditional static airburst correlations.

Figure 5 presents a comparison of blast evolution from a line source with a Chelyabinsk-like deposition profile with that of a spherical charge with a $1 \mathrm{~km}$ radius for a $90^{\circ}$ entry. The burst height for the spherical detonation was set to $29.5 \mathrm{~km}$ which corresponds closely to the half-energy altitude of the line source. Both simulations released a total of $520 \mathrm{kt}$ to facilitate ground pressure comparisons with the $18^{\circ}$ entry case presented earlier. Figure 5 presents the temporal evolution of both scenarios through several snapshots. The uppermost snapshots are taken shortly after energy release, at $t^{*}=3.14$. At the left, buoyancy-driven vortical structures travel upward along the trajectory while momentum in the core continues to advance toward the ground. At the right, the blast wave has propagated a radial distance of about $10 \mathrm{~km}$ from the blast center and the bubble has begun to flatten due to buoyancy effects acting upon the hot gas within. As the simulations advance in time, marked similarities begin to appear in the structure of the two blasts, and while the trajectory simulation retains a more intricate structure, its clear from both the color map and the location of the blast front that the strength and speed of the shock advancing toward the ground is remarkably similar in the two simulations. The final frames show the blast evolution at non-dimensional times of $\sim 34.5$ (roughly 114 seconds after detonation). Both blasts have reached the ground plane, and the ground footprint has expanded to a radius of $\sim 30 \mathrm{~km}$ from ground zero in both simulations.

Figure 6 shows a detailed comparison of the overpressure-envelope on the ground. For reference, the frame at the left shows a snapshot through the domain (via overpressure contours, $\left(p-p_{\infty}\right) / p_{\infty}$ ) taken at around 139 seconds after energy release. The right frame shows the envelope of recorded over/underpressure as well as instantaneous pressure profiles for both scenarios at 139 seconds. The yellow-shaded region shows the envelope for the spherical charge while the red line shows that of the line-source. Away from ground-zero, the envelopes for the two cases are remarkably similar, in particular the decay of the envelope with increasing radius is very well predicted by the spherical approximation. Nearer to the origin, the spherical charge is over predicts the line source however this appears to be an artifact of the particular energy deposition profile used to initiate this simulation which included a weak final fragmentation event near the end of the trajectory. Returning to Figure 5, evidence of this event is clearly visible in the top three frames of the blast from the line source and it appears to weaken the main blast front as it advances toward the ground near the origin. Since the spherical charge was initially homogeneous, it contains no such structures.

The instantaneous pressure profiles shown by the red and blue dashed lines in the frame at the right of Figure 6 show remarkable similarities. At 139 seconds, both have expanded to a radius of just over $40 \mathrm{~km}$. In addition to virtually identical propagation speeds, the two profiles show remarkably similar peak overand under pressures.

...Include follow-on discussion comparing results for spherical charge with overpressure and ground damage predictions using military-handbook correlations found in Reference [9].......

\section{Summary}

.....This work presented a new simulation technique for bolide-entry modeling using a fully-conservative, Cartesian mesh, finite-volume solver. The presentation included development of mathematical models for the deposition of mass, momentum and energy into the atmosphere and included studies for verification and validation of these models through canonical solutions of spherical blasts and the comparison of surface overpressures, and blast arrival time with actual results in the literature for the 2013 Chelyabinsk meteor.

The discussion also examined various approximations to the physics of bolide entry to decrease the computational expense while retaining sufficient fidelity to drive ground damage estimates required for credible risk assessment. We present parametric studies that quantify the influence of entry-angle, burst-height and other parameters on the ground footprint of the airburst, and these values are related to predictions from 

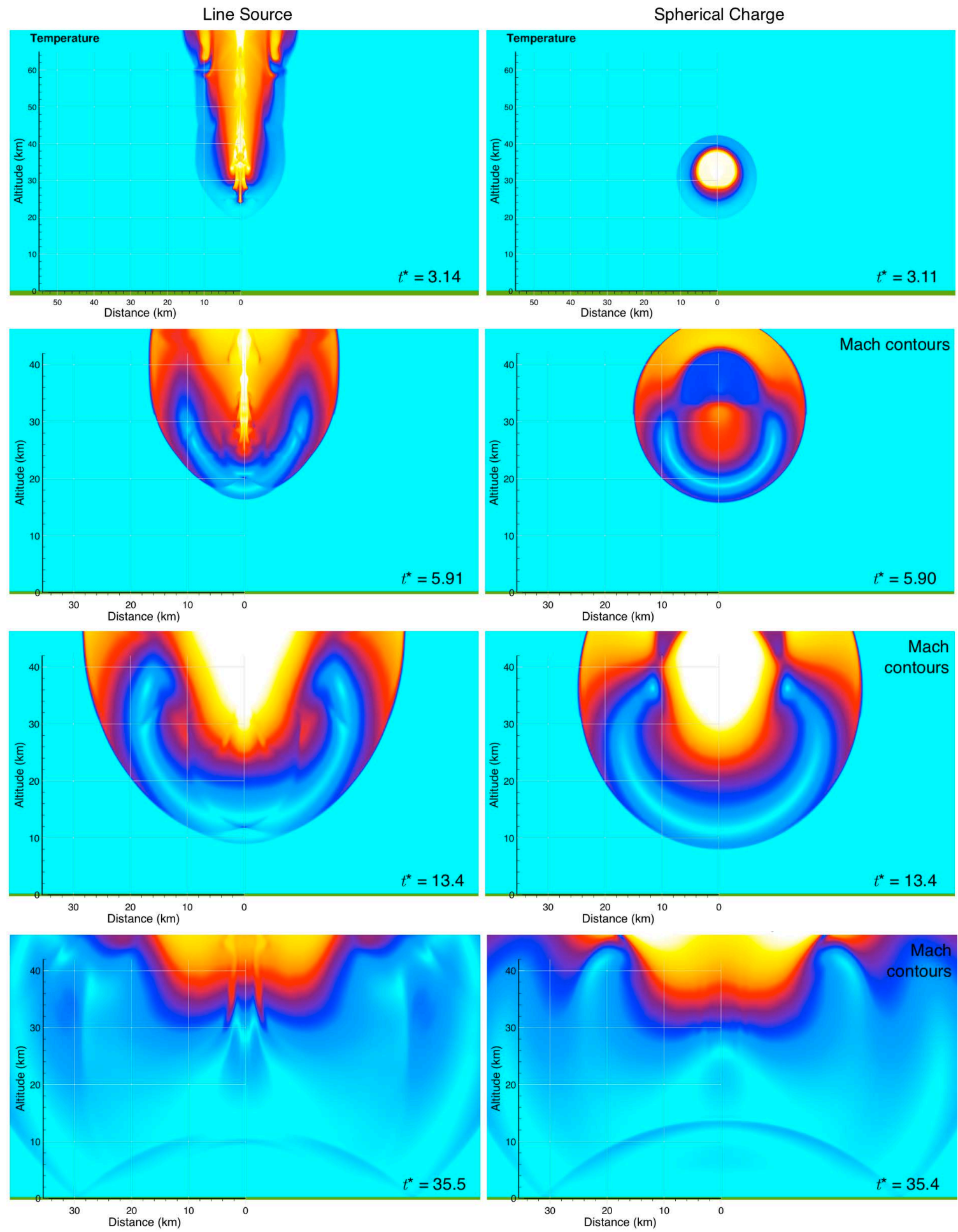

Figure 5. Comparison of blast evolution from a line source with a Chelyabinsk-like deposition profile with that of a spherical charge with a $1 \mathrm{~km}$ radius for a $90^{\circ}$ entry. Burst height $=29.5 \mathrm{~km}$, Total Energy $=520 \mathrm{kt}$, 

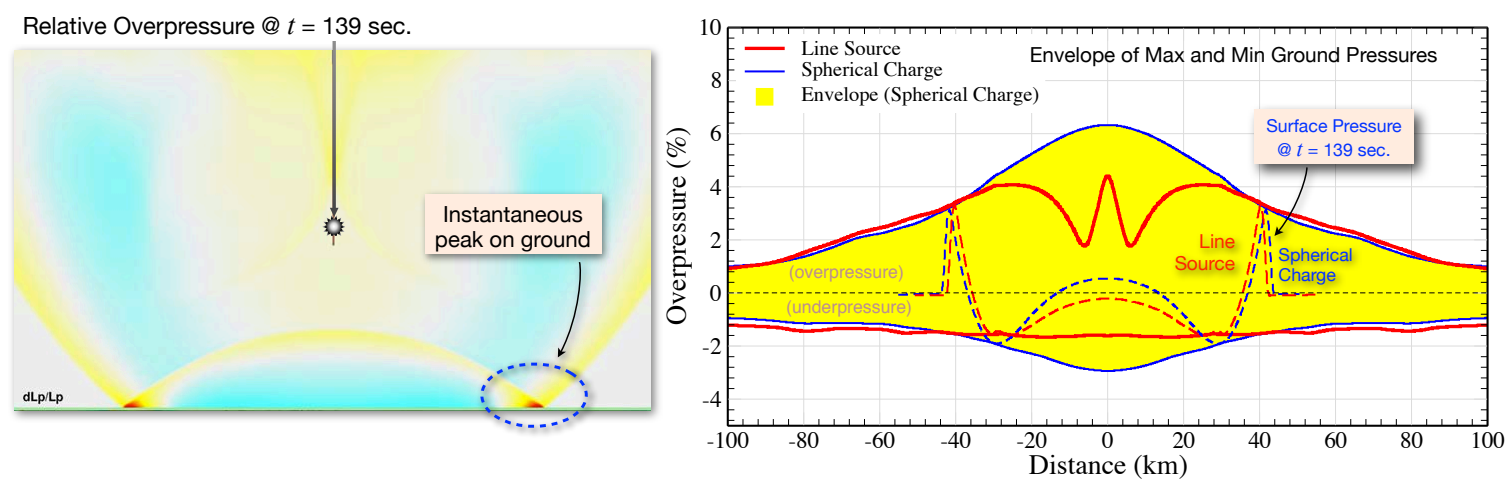

Figure 6. Comparison of overpressure envelope from a line source with a Chelyabinsk-like deposition profile with that of a spherical charge with a $1 \mathrm{~km}$ radius for a $90^{\circ}$ entry. Burst height $=29.5 \mathrm{~km}$, Total Energy $=520 \mathrm{kt}$. Dashed lines show instantaneous ground pressure profiles shown for both simulations at 139 sec after energy release.

analytic and handbook-methods. ....

\section{Acknowledgments}

The authors wish to thank the members of the NASA Ames Planetary Defense IPT for their superb effort and responsiveness throughout this work. This research was supported by the NASA Science Mission Directorate Planetary Sciences Division's Near-Earth Object program. Marian Nemec was supported under NASA Ames Research Center Contract NNA10DF26C. Computer support was provided by the NASA Advanced Supercomputing Center at NASA Ames.

\section{References}

${ }^{1}$ Olga P. Popova et al, "Chelyabinsk Airburst, Damage Assessment, Meteorite Recovery, and Characterization," Science, Vol. 342, No. 6162, 2013, pp. 1069-1073, http://dx.doi.org/10.1126/science.1242642.

${ }^{2}$ National Aeronautics and Space Administration Office of Space Science, "Bolide Events 1994-2013," http://neo.jpl.nasa.gov/news/news186.html, 2013.

${ }^{3}$ Peter G. Brown et al, "A 500-kiloton airburst over Chelyabinsk and an enhanced hazard from small impactors," Nature, Vol. 503, No. 6162, November 2013, pp. 238-241, http://dx.doi.org/10.1038/nature12741.

${ }^{4}$ Stokes et al, G. H., "Study to Determine the Feasibility of Extending the Search for Near-Earth Objects to Smaller Limiting Diameters," National Aeronautics and Space Administration Office of Space Science, August 2003, http://neo.jpl.nasa.gov/neo/neoreport030825.pdf.

${ }^{5}$ Arnold et al, J. O., "NASA Ames Planetary Defense Integrated Product Team: An Overview," Proceedings of the 12st International Planetary Probe Workshop, Cologne, Germany, June 2015.

${ }^{6}$ Shuvalov, V. and Artemieva, N., "Numerical modeling of Tunguska-like impacts," Planetary and Space Science, Vol. 50, No. 2, February 2002, pp. 181-192, http://dx.doi.org/10.1016/S0032-0633(01)00079-4.

${ }^{7}$ ReVelle, D. O., "NEO fireball diversity: energetics-based entry modeling and analysis techniques," Near Earth Objects, our Celestial Neighbors: Opportunity and Risk, Vol. 2 of Proceedings of the International Astronomical Union, 8 2006, pp. 95-106.

${ }^{8}$ Korobeinikov, V., Gusev, S., Chushkin, P., and Shurshalov, L., "Flight and fracture of the Tunguska cosmic body into the Earth's atmosphere," Comput. ES Fluids, Vol. 21, No. 3, 1992, pp. 323 - 330, http://dx.doi.org/10.1016/0045-7930(92)90041-S.

${ }^{9}$ Samuel Glasstone and Philip J. Dolan, The Effects of Nuclear Weapons, United States Department of Defense and the Energy Research and Development Administration, 3rd ed., 1977, http://www.fourmilab.ch/etexts/www/effects/.

${ }^{10}$ Boslough, M. B. E. and Crawford, D., "Low-Altitude Airbursts and the Impact Threat," International Journal of Impact Engineering, Vol. 35, No. 12, 2008, http://dx.doi.org/10.1016/j.ijimpeng.2008.07.053.

${ }^{11}$ Boslough, M. B. E., "Airburst Warning and Response," Acta Astronautica, Vol. 103, October-November 2014, pp. 370375, http://dx.doi.org/10.1016/j.actaastro.2013.09.007.

${ }^{12}$ Douglas O. ReVelle, "On Meteor-Generated Infrasound," Journal of Geophical Research, Vol. 81, No. 7, March 1976, pp. $1217-1230$.

${ }^{13}$ ReVelle, D., "Recent Advances in Bolide Entry Modeling:A Bolide Potpourri*," Earth, Moon, and Planets, Vol. 97, No. 1-2, 2005, pp. 1-35, 10.1007/s11038-005-2876-4.

${ }^{14}$ CEPLECHA, Z. and REVELLE, D. O., "Fragmentation model of meteoroid motion, mass loss, and radiation in 
the atmosphere," Meteoritics \& Planetary Science, Vol. 40, No. 1, 2005, pp. 35-54, http://dx.doi.org/10.1111/j.19455100.2005.tb00363.x.

${ }^{15}$ Hills, J. G. and Goda, P. M., "The Fragmentation of Small Asteroids in the Atmosphere," The Astronomical Journal, Vol. 105, No. 3, March 1993, pp. 1114-1144.

${ }^{16}$ Aftosmis, M. J., Berger, M. J., and Melton, J. E., "Robust and Efficient Cartesian Mesh Generation for ComponenetBased Geometry," AIAA Journal, Vol. 36, No. 6, June 1998, pp. 952-960.

${ }^{17}$ van Leer, B., "Flux-Vector Splitting for the Euler Equations," ICASE Report 82-30, Sept. 1982.

${ }^{18}$ Aftosmis, M. J., Berger, M. J., and Adomavicius, G. D., "A Parallel Multilevel Method for Adaptively Refined Cartesian Grids with Embedded Boundaries," AIAA-Paper 2000-0808, Jan. 2000.

${ }^{19}$ Aftosmis, M. J., Berger, M. J., and Murman, S. M., "Applications of Space-Filling-Curves to Cartesian methods in CFD," AIAA-Paper 2004-1232, Jan. 2004.

${ }^{20}$ Berger, M. J., Aftosmis, M. J., and Murman, S. M., "Analysis of Slope Limiters on Irregular Grids," AIAA Paper 2005-0490, Jan. 2005.

${ }^{21}$ Mannan, M. S. and Lees, F. P., Lees' Loss Prevention in the Process Industries (Third Edition), Butterworth-Heinemann, Burlington, third edition ed., 2005.

${ }^{22}$ Mathias, D. L., Robertson, D. K., and Aftosmis, M. J., "Sensitivity of Ground Damage Prediction to Meteoroid Breakup Modeling Assumptions." 4th IAAA Planetary Defense Conference (PDC 2015), 2015. 